\title{
Nicholas Olenev
}

Russian Academy of Sciences, Dorodnicyn Computing Centre, Moscow, Russia

\section{A NORMATIVE DYNAMIC MODEL OF REGIONAL ECONOMY FOR STUDY ECONOMIC INTEGRATIONS}

Key words: Dynamic model, regional economy, economic integration

\section{$\underline{A B S T R A C T}$}

A simplest multi-sector dynamic model for regional economy is a normative balance mathematical model, but it contains a lot of unspecified parameters which are not defined directly by the data of economic statistics. Only confidence intervals for the unknown parameters can be computed from the statistical data. This work presents a method for estimation of the model parameters by application of parallel computations on multi-processors systems and by some heuristic algorithms. They determine the unknown parameters of economic model by indirect way, comparing time series for macro indexes calculated by model with statistical time series for these indexes. A new wavelet based measure of similarity was used. The use of the method is illustrated by the parameter estimation of a macroeconomic model of Kirov (Vyatka) Region of Russia for 2000-2006. The each production sector shadow money stock grows due to sale of shadow final product to households and as intermediate product to other sectors. Calibrated model is used for estimation of the Regional Government economic politics in particular for politics of regional economic integration.

\section{INTRODUCTION}

Parallel calculations on modern multiprocessors computers open new opportunities in application of mathematical models for research of regional transition economy, including processes of economic integration. Application of normative economic models was limited by complexity of parameter estimation for lot of unknown parameters in such models. This work offers a technique for economic model parameter estimation and presents criteria of closeness and similarity of time series for economic parameters. They use these criteria for indirect estimation of unknown economic model parameters by comparison of calculated macroindexes with their statistical analogues. The simulation model of regional economy enables to receive a quantitative estimation of dynamics of macroindexes for regional economy.

It is possible to use various types of mathematical models of multisector regional economic systems for the quantitative analysis of economic development: from inputoutput model to the models constructed on the description of hierarchy for optimization 
problems of chosen economic agents. However, the simplest model for description of transition processes is a normative model.

It is required much less time for construction of normative balance dynamic models then for dynamic models based on solution of optimal problems. The three sector normative balance mathematical model of the Kirov Region economy considers the taxation and a shadow turnover. Dynamics of material and financial balances of the model is set by change of product stocks, production factors and money. This regional model has eight economic (representative) agents: three producers (Production sectors $X$. $Y, Z$ ), Households $(H)$; Regional bank system $(B)$, Trade intermediary $(T)$, Government $(G)$, Outside world $(O)$.

Production sectors in the regional economic model are presented by three sectors: $(X)$ the timber industry complex including forestry; $(Y)$ a complex of new innovation industries in biotechnology and the chemistry, including science and education; $(Z)$ combination of the remained industries.

The each production sector official stock of money grows due to credits, due to sale of the goods on a outside market and in the inner markets, due to transfers from the budget and receipts of the washed money from a shadow turn. It decreases due to payment to households, intermediate consumption, credits payments and transfers of taxes to the consolidated regional budget.

However the model contains about hundred equations and more than eighty parameters which cannot be defined directly from statistical data. It would seem that normative models of such type are absolutely senseless, as for carrying out of the intelligent qualitative and quantitative calculations on them it is necessary to estimate their external parameters, but the most part of the parameters cannot be estimated directly from economic statistical data. Even in a case when we have all statistical data the quality of them is as a rule such that they suffice only for determination of intervals for the model parameters. Besides, the initial values of some model variables are unknowns and consequently should be considered as such parameters as well. Moreover, even if all necessary statistics is available the quality of the data isn't always good.

Here the new technology of estimation for external parameters of the model is offered. It based on use of high-speed parallel calculations on multiprocessing systems. For parameter estimation of the model time series of regional economic macroindexes calculated by the model are compared to corresponding statistical time series of them. As criteria of affinity of time series the Theil index of discrepancy and recently constructed similarity criterion for nonlinear time series based on wavelet coefficients are used.

At construction of the model and its parameter estimation they solve problems of the analysis and structurization of initial data in conformity with model structure. So, at time of model construction the problem of a choice and the system analysis of initial statistical data is solved. Conclusions and the forecasts received by scenario calculations on simulation model basically can be experimentally denied, and 
hypotheses on which the model is based, can be challenged. It gives the opportunity to modify model in future and receive more proved forecasts by constantly improved model and make more proved analytical conclusions.

This paper gives an introduction to mathematical modelling of regional economy by normative balance dynamic model, and present an application of the technique to the analysis of economic integration The main purpose of the paper is to illustrate the method, rather than present a state-of-the-art analysis. Math modelling approach makes it possible to pursue the analysis further than possible with analytic methods, and it can yield qualitative as well as quantitative results. A model is presented, and it is used to analyse the consequences of integration processes: influence of custom tariffs reducing on region welfare.

\section{MODEL}

Regional economy production sectors $(X, Y, Z)$ use labour, the capital and intermediate products of adjacent production sectors. Production sectors deliver product on domestic market, on outer market, and on the market of intermediate product. It is considered that the prices are formed in each market of each product and change of the prices is in inverse proportion to change of stocks of corresponding products. Households $L$ offer labour and consume final production. Trading intermediary $T$ redistributes material and financial flows. Bank system $B$ emits money resources, gives out credits to production sectors. The regional government $G$ accumulates taxes from production sectors (the profit tax $n_{1}$, the value-added tax $n_{2}$ and excises $n_{3}$, the uniform social tax $n_{4}$, the customs duties on export $n_{5}$ ) and households (the customs duties on import $n_{6}$, the $\operatorname{surtax} n_{7}$ ) and adjusts charges of the budget.

To account of a shadow turnover in model, we assume, that production sectors divide the product on official and shadow one and that the last is not taxed. As a result each production sector has two kinds of money - "white" and "black". "Black" money can be washed, and the stock of not washed money is exposed to penal sanctions ("taxation" of shadow sector). All money of the consumer are considered "white", and the consumer divides the income by norms of consumption of official and shadow products of all sectors.

For simplicity standard designations will be used. Macroindexes and parameters of the model are supplied by the top and bottom indexes, and the top indexes are used to point agents, and bottom to point the goods. Thus the same symbols can be used as top or bottom index or can be stayed at normal place but they designate the different things. In the given work parameters and intensive indexes will be designated by small letters, and extensive macroindexes and the called indexes by capital letters. It is considered that distribution of a stock of each good is made by norm: $a_{i}^{n m}$ - a share of a stock of the good $i$ going from economic agent $n$ to economic agent $m\left(a_{i}^{n n}=a_{i}^{n}\right)$. It is considered that distribution of money is made also under some norm: $b_{i}^{m n}$ - a share of money stock going from agent $m$ to agent $n$ for a product $i\left(b_{i}^{n n}=b_{i}^{n}\right)$. Capital intensities also are set by some norms: $c_{i}^{m}$ - agent $m$ norm of expenses on product $i$ for creation of one unit of capital product. Parameters of production functions of sectors 
are set by constants. For example, output $Y_{X}(t)$ of product $X$ produced by economic agent $X$ (timber industry complex of the Kirov Region, sector $X$ ) is described by CobbDouglas production function of used production factors (stocks $Q$ ): labour $L$, capital $K$ and intermediate products from sectors $Y$ and $Z$.

$$
Y_{X}=\left(a_{L}^{X} Q_{L}^{X}\right)^{\delta_{L}^{X}} \cdot\left(a_{K}^{X} Q_{K}^{X}\right)^{\delta_{K}^{X}} \cdot\left(a_{Y}^{X} Q_{Y}^{X}\right)^{\delta_{Y}^{X}} \cdot\left(a_{Z}^{X} Q_{Z}^{X}\right)^{\delta_{Z}^{X}}
$$

where $\delta_{L}^{X}+\delta_{K}^{X}+\delta_{Y}^{X}+\delta_{Z}^{X}=1$. Hereinafter, all parameters $Y_{X}(t), Q_{L}^{X}(t), Q_{K}^{X}(t)$, $Q_{Y}^{X}(t), Q_{Z}^{X}(t)$, are considered as functions of time $\mathrm{t}$, therefore this argument falls in formulas. All parameters, as a rule, are considered as constants, as here $a_{L}^{X}, a_{K}^{X}, a_{Y}^{X}$, $a_{Z}^{X}, \delta_{L}^{X}, \delta_{K}^{X}, \delta_{Y}^{X}, \delta_{Z}^{X} \in(0,1)$. Agent $X$ produces official product $X$ and shadow product $V$ by the common capital, the common labour and the common stocks of intermediate products, and made product (output) $Y_{X}(t)$ shares in fix proportion $\left(1-q_{X}\right): q_{X}$ on open ("white") output $X$ and shadow ("black") output $V$, where $q_{X}$ is a shadow share in the common output of the product $Y_{X}(t)$. The shadow product is used for sale to population and other sectors. It is assumed in the model that investments can be official only. The stock of open product $Q_{X}{ }^{X}(t)$ increases due to production and decreases due to shipment to agents $Y, Z, L$ (ships to the intermediary by the norm) and for investments $I_{X}(t)$. It is considered that charges on investments from the own product coincide with incomes of them. There is a fix share of own product stock that goes on outer market $X_{X}{ }^{X O}(t)$.

$$
\frac{d Q_{X}^{X}}{d t}=\left(1-q_{X}\right) Y_{X}-\left(a_{X}^{X L}+a_{X}^{X Y}+a_{X}^{X Z}+a_{X}^{X O}\right) Q_{X}^{X}-c_{X}^{X} I_{X}
$$

where

$$
I_{X}=\frac{b_{K}^{X} W^{X}}{p_{X}^{X} c_{X}^{X}+p_{Y}^{X} c_{Y}^{X}+p_{Z}^{X} c_{Z}^{X}}, \quad p_{X}^{X}=\min \left\{p_{X}^{Y}, p_{X}^{Z}\right\}, \quad X_{X}^{X O}=a_{X}^{X O} Q_{X}^{X}
$$

The shadow share $q_{X}$ in total output defines a gain of stocks both opened $Q_{X}{ }^{X}(t)$ and shadow $Q_{V}{ }^{X}(t)$ products. The stock of shadow product $V$ in sector $X$ decreases due to deliveries to households and allied sectors.

$$
\frac{d Q_{V}^{X}}{d t}=q_{X} Y_{X}-\left(a_{V}^{X L}+a_{V}^{X Y}+a_{V}^{X Z}\right) Q_{B}^{X}
$$

Change of intermediate products' stocks for sector $X$, change of labour and capital stocks is similarly described.

The stock of open ("white") money $W_{X}(t)$ of economic agent grows when agent $X$ takes bank credits, sales the goods on outer market, sales the goods on domestic markets, takes transfers from regional consolidated budget $T^{G X}(t)$ and receipts of the "washed" money from a shadow turnover $b_{B}{ }^{X} B^{X}(t)$. It decreases due to wages payments to 
households $L$, due to consumption of intermediate products of adjacent sectors $Y$ and $Z$, due to payments on credits and transfers of taxes to the consolidated budget.

$$
\begin{aligned}
& \frac{d W^{X}}{d t}=w p_{X}^{O} X_{X}^{X O}+C^{B X}+\left(p_{X}^{L} a_{X}^{X L}+p_{X}^{Y} a_{X}^{X Y}+p_{X}^{Z} a_{X}^{X Z}\right) Q_{X}^{X}- \\
& -\left(b_{Y}^{X Y}+b_{Z}^{X Z}+b_{W}^{X Y}+b_{U}^{X Z}+b_{L}^{X L}+b_{H}^{X B}\right) W^{X}-T^{X G}+T^{G X}+b_{B}^{X} B^{X},
\end{aligned}
$$

where $w(t)$ is Ruble/USD exchange rate, $T^{X G}(t)$ is transfer payments to the consolidated regional budget (equal the sum of taxes), $T^{G X}(t)$ is transfer from the budget to sector (VAT returns plus grants and so on). Deductions of agent $\mathrm{X}$ to the consolidated budget $T^{X G}(t)$ develop from the profit tax $T_{1}^{X G}(t)$, the added value tax $T_{2}^{X G}(t)$, the excises $T_{3}^{X G}(t)$, the uniform social tax $T_{4}^{X G}(t)$, to customs duties on export $T_{5}{ }^{X G}(t)$.

$$
T^{X G}=T_{1}^{X G}+T_{2}^{X G}+T_{3}^{X G}+T_{4}^{X G}+T_{5}^{X G},
$$

where

$$
\begin{aligned}
& T_{5}^{X G}=n_{5} w p_{X}^{O} X_{X}^{X O}, \quad T_{4}^{X G}=n_{4} b_{L}^{X L} W^{X}, \\
& T_{3}^{X G}=n_{3}^{X}\left[w p_{X}^{O} X_{X}^{X O}+\left(p_{X}^{L} a_{X}^{X L}+p_{X}^{Y} a_{X}^{X Y}+p_{X}^{Z} a_{X}^{X Z}\right) Q_{X}^{X}\right], \\
& T_{2}^{X G}=n_{2}\left\{w p_{X}^{O} X_{X}^{X O}+\left(p_{X}^{L} a_{X}^{X L}+p_{X}^{Y} a_{X}^{X Y}+p_{X}^{X Z}\right) Q_{X}^{X}-\right. \\
& \left.\left(b_{Y}^{X Y}+b_{Z}^{X Z}+b_{H}^{X B}\right) W^{X}-T_{3}^{X G}-T_{4}^{X G}-T_{5}^{X G}\right\}, \\
& T_{1}^{X G}=n_{1}\left\{w p_{X}^{O} X_{X}^{X O}+\left(p_{X}^{L} a_{X}^{X L}+p_{X}^{Y} a_{X}^{X Y}\right) Q_{X}^{X}-\right. \\
& \left.-\left(b_{Y}^{X Y}+b_{Z}^{X Z}+b_{H}^{X B}+b_{L}^{X L}\right) W^{X}-T_{2}^{X G}-T_{3}^{X G}-T_{4}^{X G}-T_{5}^{X G}\right\} .
\end{aligned}
$$

The stock of shadow money grows by sale of a shadow product as final product to households $L$ and, as intermediate product, to adjacent sectors $Y$ and $Z$, a part $b_{B}{ }^{X}$ of shadow money stock has time to wash, a part $b_{B}{ }^{X G}$ gets as penal sanctions in a profitable part of the regional government consolidated budget, and a part $b_{B}{ }^{X L}$ goes to the households as shadow incomes.

$$
\frac{d B^{X}}{d t}=\left(p_{V}^{L} a_{V}^{X L}+p_{V}^{Y} a_{V}^{X Y}+p_{V}^{Z} a_{V}^{X Z}\right) Q_{V}^{X}-\left(b_{B}^{X L}+b_{B}^{X}+b_{B}^{X G}\right) B^{X} .
$$


It is considered that agent $X$ takes all the credit offered by regional bank system $\mathrm{B}$, however the volume of given credit $C^{B X}(t)$ is limited by liquidating cost of production capital which is considered proportional the capital stock: $C^{B X}=\sigma^{X} Q_{K}^{X}, \sigma^{X}>0$. Debts $Z^{X}(t)$ of agent $X$ to bank system $B$ grows due to delivery of new credits $C^{B X}(t)$ and charges of current interest rate $r(t)$ on an available debts, and decreases due to repayment flow $H^{X B}(t)$.

$$
\frac{d Z^{X}}{d t}=C^{B X}+r Z^{X}-H^{X B}, \quad H^{X B}=b_{H}^{X B} W^{X} .
$$

Corresponding equations for sectors $Y$ and $Z$ are easy write by analogy to equations (1) - (12) for sector X.

Change of stock for final product $X$ of timber industry complex of the Kirov Region, intended to agent $L$ (households), $Q_{X}{ }^{L}(t)$ defines change of a consumer price index $p_{X}{ }^{L}$ on product $X$.

$$
\begin{aligned}
\frac{d Q_{X}^{L}}{d t} & =a_{X}^{X L} Q_{X}^{X}-\frac{b_{X}^{L X} W^{L}}{p_{X}^{L}}, \\
\frac{d p_{X}^{L}}{d t} & =\alpha_{X}^{L}\left(\frac{b_{X}^{L X} W^{L}}{p_{X}^{L}}-a_{X}^{X L} Q_{X}^{X}\right) .
\end{aligned}
$$

Changes of stocks of all other products in all markets and change of the corresponding prices are similarly defined. Thus we assume, that the product of the same sectors acts in other quality in the different markets and has the other price, that it is, as a matter of fact, other product.

It is considered that increase of the open and shadow wages can occur both at shortage of the labour, and due to increase of consumer prices on production of the sector. For example, the open wage at sector $X$ is determined by equation

$$
\frac{d s_{L}^{X}}{d t}=\left[\alpha_{L}^{X}\left(\frac{b_{L}^{X L} W^{X}}{s_{L}^{X}}-a_{L}^{L X} Q_{L}^{L X}\right)+\frac{\beta_{L}^{X} s_{L}^{X}}{p_{X}^{L}}\left(\frac{b_{X}^{L X} W^{L}}{p_{X}^{L}}-a_{X}^{X L} Q_{X}^{X}\right)\right]_{+}
$$

Here the following designation is used: $A_{+}=A$, if $A>0$ and $A_{+}=0$, if $A \leq 0$. It is supposed that the gain of wages at surplus of a manpower does not exceed a gain of the price, $\beta_{L}^{X} \leq \alpha_{X}^{L}$.

The stock of money on the regional consolidated budget accounts increases by tax revenues and decreases by transfers to production sectors and households. 


$$
\begin{aligned}
& \frac{d W^{G}}{d t}=b_{V}^{X G} B^{X}+b_{W}^{Y G} B^{Y}+b_{U}^{Z G} B^{Z}+T^{X G}+T^{Y G}+T^{Z G}+T^{L G}- \\
& -\left(b_{X}^{G X}+b_{Y}^{G Y}+b_{Z}^{G Z}+b_{L}^{G L}\right) W^{G} .
\end{aligned}
$$

The regional bank system is not closed, and the branches of the Russian banks from other regions play greater role in investment decisions. Therefore as the first step to description of considered regional bank system it was considered that a part gold and exchange currency reserves of the Russian Federation provide reservation for actives of the region.

The description of the model terminates on it because of restrictions on volume.

\section{PROBLEM OF PARAMETER ESTIMATION FOR ECONOMIC MODEL}

In this work a method for macroeconomic model parameter estimation based on parallel processing is proposed. For an application of this method some measure of similarity between two time-series is required and so a new wavelet based measure of similarity was elaborated. Complicated economic models with a lot of parameters can be accurately estimated by the proposed method. The use of the method is illustrated by the parameter estimation of a regional macroeconomic model.

A macroeconomic model usually contains a lot of unspecified parameters. Some variables of the macroeconomic model have initial values that are sometimes unknown and should be considered as parameters as well. In most cases mentioned parameters can't be defined on the basis of economic statistics. Moreover, even if all necessary statistics is available the quality of the data isn't always good. That's why only confidence intervals for the unknown parameters can be computed from the data.

For estimation of the unknown parameters time-series for some macro-indexes (calculated by the model) and statistical time-series for these macro-indexes should be compared by means of some measure of similarity. The unknown parameters can be determined implicitly as those parameters, which provide minimum value of the used measure of similarity. Parallel processing on a cluster of workstations or on a supercomputer enables to perform exhaustive search of the parameters within their confidence intervals (determined either from economic sense or from the available statistical data) and estimate their values for reasonable time. In order to use parallel processing the macroeconomic model should be divided into blocks so that parameter estimation of any block can be done independently of the other blocks. In this case not only real statistical data, but also data calculated from some block can be used as values for external variables of the other blocks.

Estimated values of the parameters give new knowledge about the macroeconomic system under investigation and this knowledge can force a researcher to modify the model. Thus macroeconomic model parameter estimation based on parallel processing becomes a powerful tool for mathematical modelling of economic systems. 
It was mentioned that calculated and statistical time-series for some macro-indexes should be compared on the basis of some measure of similarity. As used here, two time-series are considered to be similar if they are close as functions of time (in other words, if there is a strong, possibly nonlinear dependence between two time-series). Convolution of the Theil's index and specially designed wavelet based measure of similarity was used as a characteristic of closeness between two time-series.

With linear trend appropriate rescaling with respect to ordinate axe two completely different time-series can become quite similar and the value of Euclidian distance between these time-series can decreases significantly. Such effect frequently occurs in many real situations.

Thus the similarity between two time-series should be based on certain characteristics of these time-series rather than on the raw values. These characteristics should be calculated in linear time, robust for changes in level, scale and trend of the time-series and the similarity between two time-series is simply given by the (traditional) similarity of the corresponding characteristics.

It is proposed to compute such characteristics on the basis of discrete wavelet transform. In that case these characteristics have all necessary properties. It is recommended to use Daubechies wavelet and scaling filters. If that's the case wavelet coefficients are robust for linear trend. That is if the time-series of a macro-index is simply a linear function of time, all wavelet coefficients are zero.

The described approach of exhaustive search for unknown parameters enables to find the solution, however time of calculations is rather big. A reduction of computation time can be achieved by means of special computation algorithms.

Macroeconomic model should be divided into blocks so that parameter estimation of any block can be done independently. Confidence intervals for parameters should be determined either from economic sense or from statistical estimation based on the real data.

Let $\widetilde{W}_{j, k}^{X}$ and $\widetilde{W}_{j, k}^{Y}$ are vectors of spectral characteristics of time series of identical length $X$ and $Y$ accordingly. As a measure of affinity between time series it is possible to use cosines of angle between vectors of characteristics, namely

$$
D(X, Y)=\cos \alpha=\left|\sum_{j, k} \widetilde{W}_{j, k}^{X} \cdot \widetilde{W}_{j, k}^{Y}\right|
$$

The introduced similarity index $D(X, Y)$ characterizes how much strong is nonlinear dependence between time series $X$ and $Y$ (in other words, it shows how much fluctuations of time series $X$ and $Y$ are similar in relation to their linear trends). The similarity index is a measure of force and an communication orientation between compared time series and, the more it is closer to unit, the more similar is behaviour of these series. 
The Theil E (X. Y) measures discrepancy of time series $X_{t}$ and $Y_{t}$ and the more close it to zero, the more close compared numbers. For convenience of calculations, instead of Theil index we shall use an affinity index $U(X, Y)=1-E(X, Y)$.

$$
U(X, Y)=1-\sqrt{\frac{\sum_{t=t_{0}}^{T}\left(X_{t}-Y_{t}\right)^{2}}{\sum_{t=t_{0}}^{T} X_{t}^{2}+\sum_{t=t_{0}}^{T} Y_{t}^{2}}} .
$$

Decomposition of model on separate blocks enables define independent parameters for reasonable time due to parallel calculations for model parameter estimation on the fixed intervals of their changes.

For unambiguity of choice for optimal point it is possible to use some convolution of affinity and similarity indexes for time series of macroindexes. For example, if for all macroparameters the adjustment of estimated by model and statistical data has about equal importance, it is possible to maximize compound value of all indexes.

Recording formal

$$
K(\vec{a}) \rightarrow \max _{\vec{a} \in A}
$$

where

$$
\begin{aligned}
& A=\left\{\vec{a} \in R^{N}: a_{i}^{-} \leq a_{i} \leq a_{i}^{+}, 1 \leq i \leq N\right\}, \\
& K=\sqrt[2 m]{\prod_{j=1}^{m} D_{j}(\vec{a}) U_{j}(\vec{a})} .
\end{aligned}
$$

Here $m$ is a total number of macroindexes; $j=1, \ldots, m$.

Thus it is necessary to touch only those variants of values of parameters, at which affinity and similarity indexes are above some fix positive values, for example, $D_{j}>0.7, U_{j}>085(j=1, \ldots, m)$.

Now full search on all unknown parameters, set on an interval, even on the most powerful supercomputers is impossible, as search on a grid from 10 points on each interval on 80 parameters gives $10^{80}$ variants, that approximately is equal to number of nucleons in the Metagalaxy. Means it is necessary to use the directed search, to reduce number of independent parameters due to additional assumptions and to use decomposition of model on rather independent blocks, identification of parameters in which can be made independently, using fixed parameters of other blocks. 
For reduction of number of independent parameters we shall define parameters of the production functions separately on the basis of statistical data and besides we shall exclude a part of parameters due to the assumption about general equilibrium fot initial date. It was possible to reduce number of independent parameters down to 9 if the similar by economic sense parameters of production sectors are equaled to each other.

The efficient variant of model parameters in which model parameters are identified under the order of value. Calculations on supercomputers of Joint Super Computer Centre and Dorodnicyn Computing Centre of the Russian Academy of Sciences and in distributed environment (GRID) were made with nine independent parameters.

\section{NUMERICAL EXPERIMENTS WITH MODEL}

Numerical experiments with model were spent to find the efficient variant qualitatively truly reflecting processes, occuring in economy of the Kirov Region. Numerical experiments have shown working effectiveness of full model and its separate parts (Figure 1). It means that the model can be used in the further work. External parameters of this variant can be taken for a basis for more exact identification of parameters of model in the future, and to use the variant as base of numerical qualitative scenario calculations with model.

Figure 1 Production outputs in production sectors of Kirov Region (Y_X - output of sector $X, Y_{-} Y$ - output of sector $Y, Y_{-} Z$ - output of sector $Z$ )

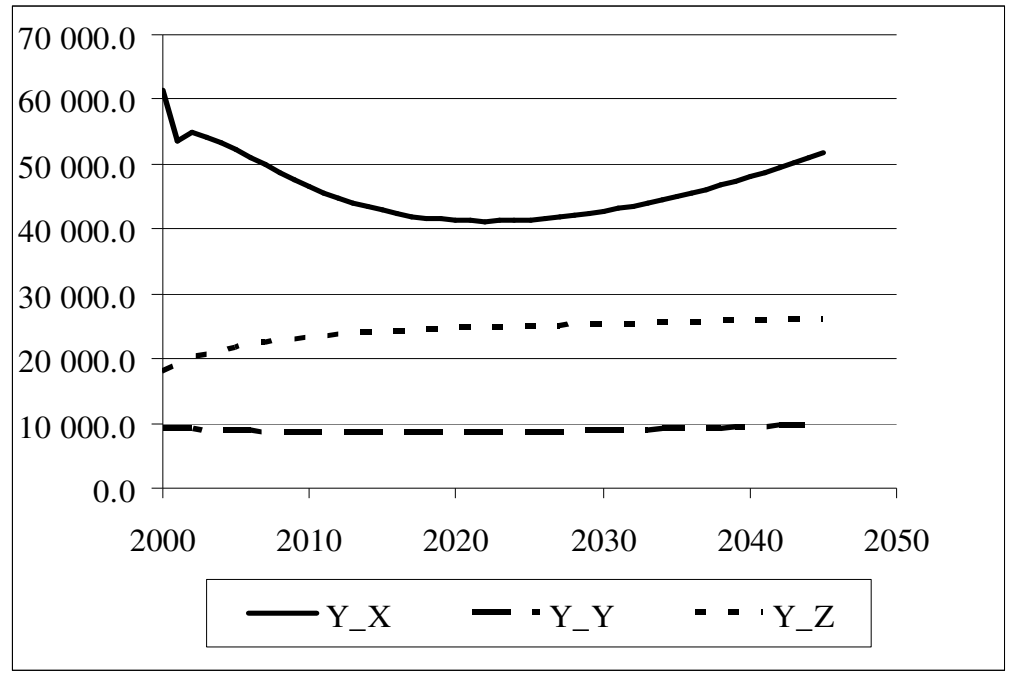

Source: estimations by model

Changes in scenario calculation in comparison with the base variant can be represented by a variation of change of the macroindexes expressed in percentage. If $F(t)$ is a value 
of some macroindex at moment of time $t$ in the base variant, and $S(t)$ is a value of the same macropindex in the current scenario, variation $V(t)$ for macroindex changes is defined as $V(t)=100 \% \cdot(S(t) / F(t)-1)$.

Presented here investigation of economic integration by model of regional economy consists of only one scenario (Figures 2-8). The scenario studies the influence of economic integration on customs duties: custom duties of production sectors on export $n_{5}$ and custom duties of households on import $n_{6}$ reduce in model approximately twice from the beginning of year 2008 (In scenario numerical experiment with the model of Kirov Region economy custom duties on export $n_{5}$ reduces from 0.12 to 0.08 and custom duties on import $n_{6}$ reduces from 0.17 to 0.08 ).

Figure 2 Variations for outputs of regional production sectors ( $Y \_X$ - output of sector X, Y_Y - output of sector Y, Y_Z - output of sector Z)

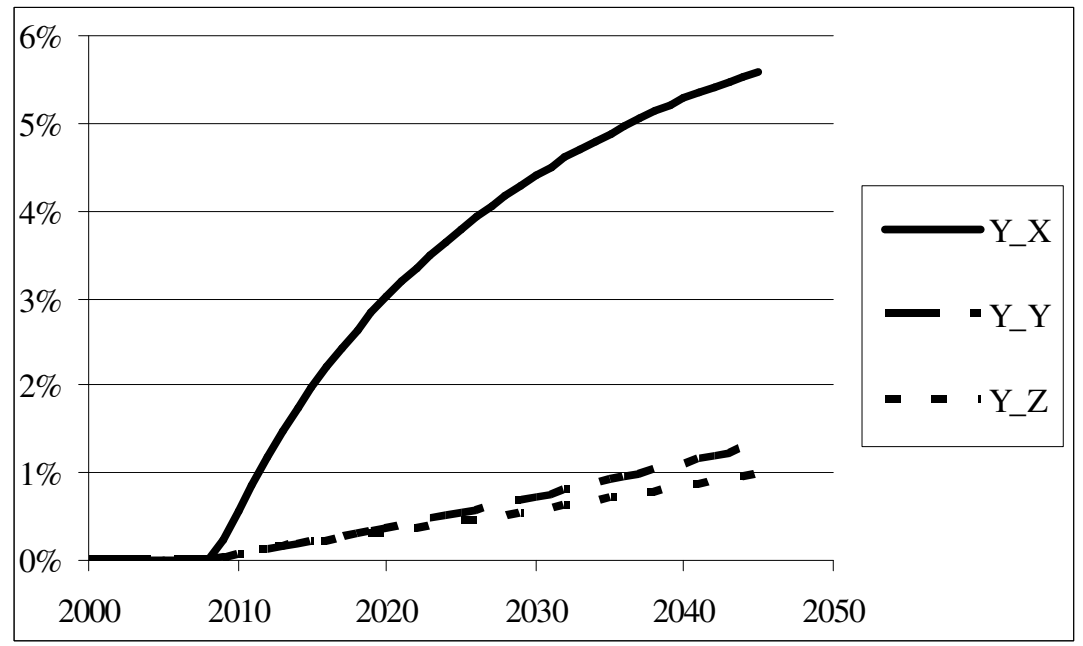

Source: estimations by model II

For Figure 2 one can see that these changes give increase for all outputs of three production sectors of Kirov Region economy: output of export oriented sector $\mathrm{X}$ increased on $5.5 \%$, but also increased on $1 \%$ the outputs for sectors $Y$ and $Z$ dependent on import concurrency. It is connected with increase of demand for intermediate production from sector $X$. 
Figure 3 Variations for capitals of regional production sectors $\left(Q K \_X\right.$ - capital of sector X, QK_Y - capital of sector $Y, Q K \_Z$ - capital of sector $Z$ )

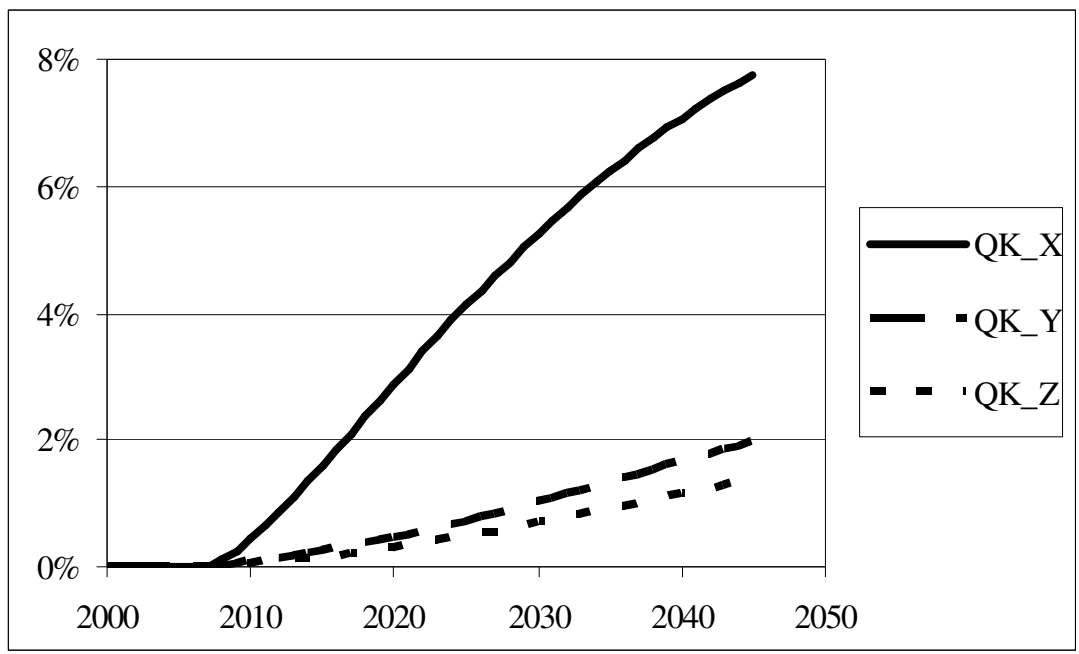

Source: estimations by model $\mathbb{\|}$

Figure 3 show the increase of capital in all production sectors.

Figure 4 Variations for price indexes on product $Z$ markets $\left(p Z \_Y\right.$ - price index on $Z$ for sector $Y, p Z \_X$ - price index on $Z$ for sector $X, p Z \_L$ - price index on $Z$ for households $\mathrm{L}$ )

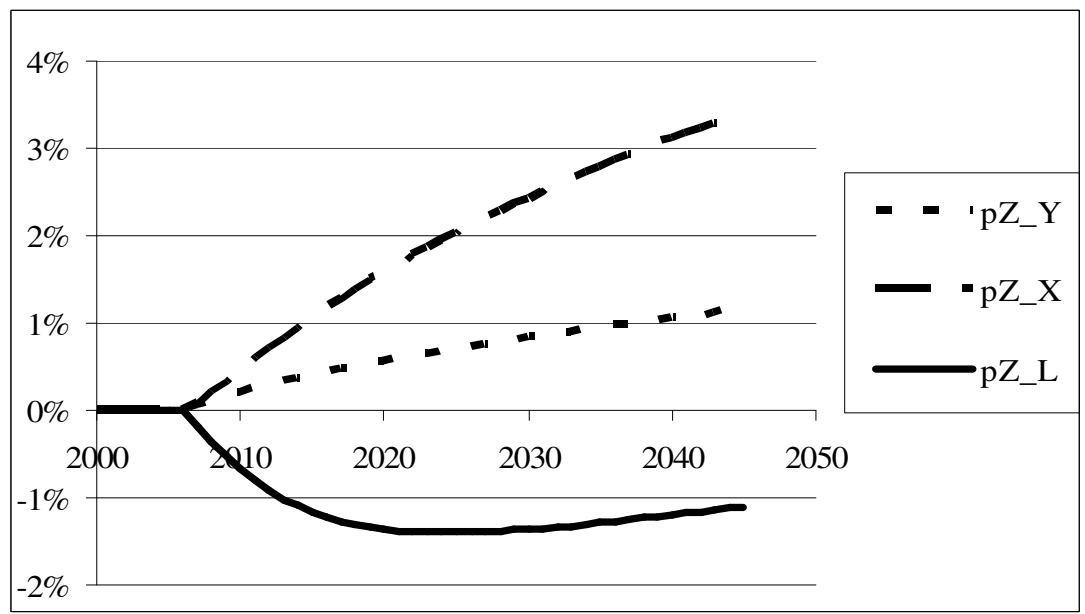

Source: estimations by model II 
In Figure 4 one can see that the price indexes vary on a miscellaneous in the different domestic markets. Price index for households in scenario of integration is decreased on $1.5 \%$ in comparison with a base variant of calculation and price indexes of intermediate products for sectors are increased. In the Figure 4 one can see that price index on product $Z$ for sector $X$ is increased twice more than price index on product $Z$ for sector $Y$. Note that changes of price indexes on outer markets are not considered in the scenario.

Figure 5 Variations for wage indexes at production sectors ( $\mathbf{S L} \mathbf{X}$ - wage index for formal part of labour force at sector $X, s L \_V$ - wage index for informal part of labour force at sector $X, s L_{-} Y$ - wage index for formal part of labour force at sector $Y$, sL_W - wage index for informal part of labour force at sector $Y, s L \_Z$ wage index for formal part of labour force at sector $\mathrm{Z}$, $\mathbf{s L} \mathbf{U}$ - wage index for informal part of labour force at sector $\bar{Z}$ )

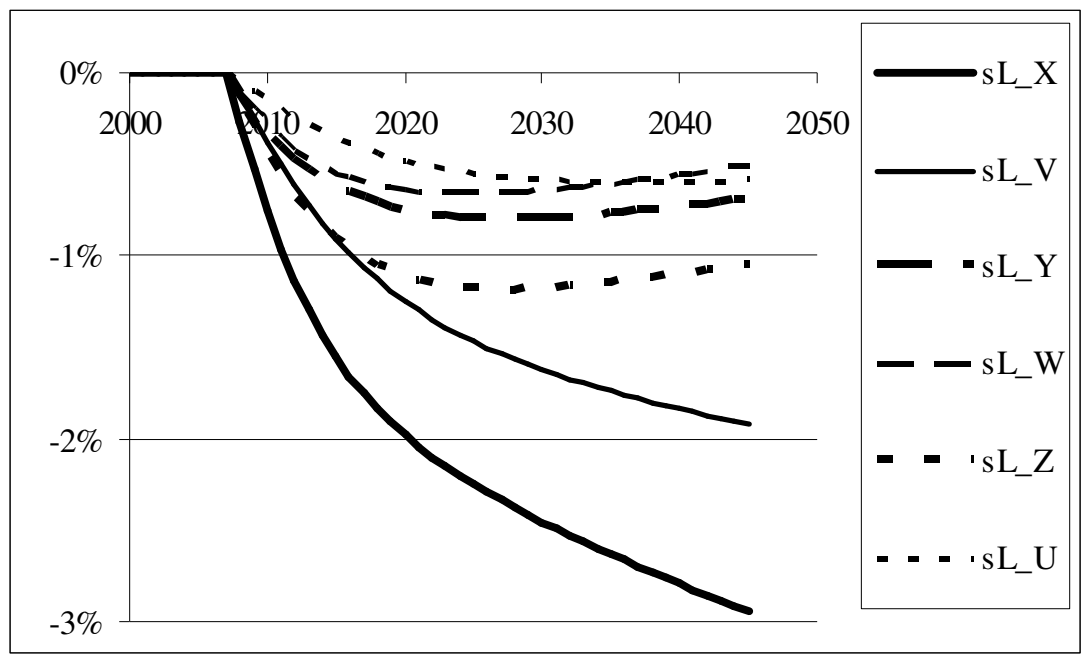

Source: estimations by model II

The decrease of consumer price indexes conducts to decrease in all wage indexes on all channels of realization: both on official and on shadow ones (Figure 5). From Figure 5 one can see that the wage indexes for shadow channels decrease not so much as them do for official channels, and differently by sector: the more big decrease is for export sector $X$ (official wage for official product $X$ and shadow wage for informal product $V$ ), less for innovative sector $Y$ (official wage for official product $Y$ and shadow wage for informal product $W$ ), and less for sector $Z$ (official wage for official product $Z$ and shadow wage for informal product $U$ ). 
Figure 6 Variations for money of regional production sector $\mathrm{X}$ (WX - white money of sector $\mathrm{X}, \mathrm{BX}$ - black money of sector $\mathrm{X}$ )

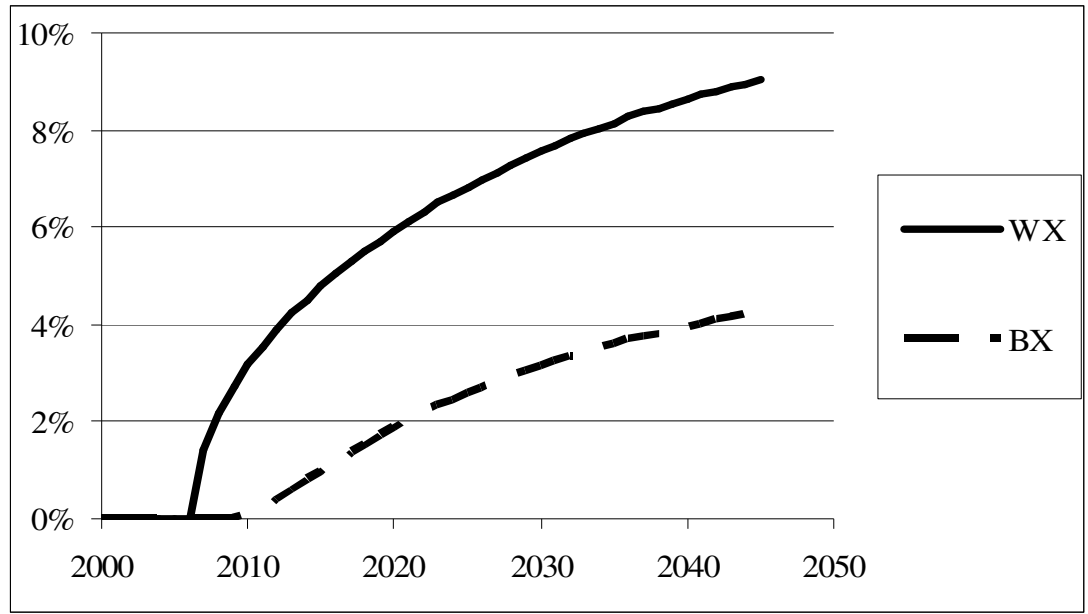

Source: estimations by model II

Black and white money stocks of sectors are increased.

Figure 7 Variations for regional government budget income from production sectors (TXG - regional government budget income from sector X, TYG regional government budget income from sector $Y$, TZG - regional government budget income from sector $Z$ )

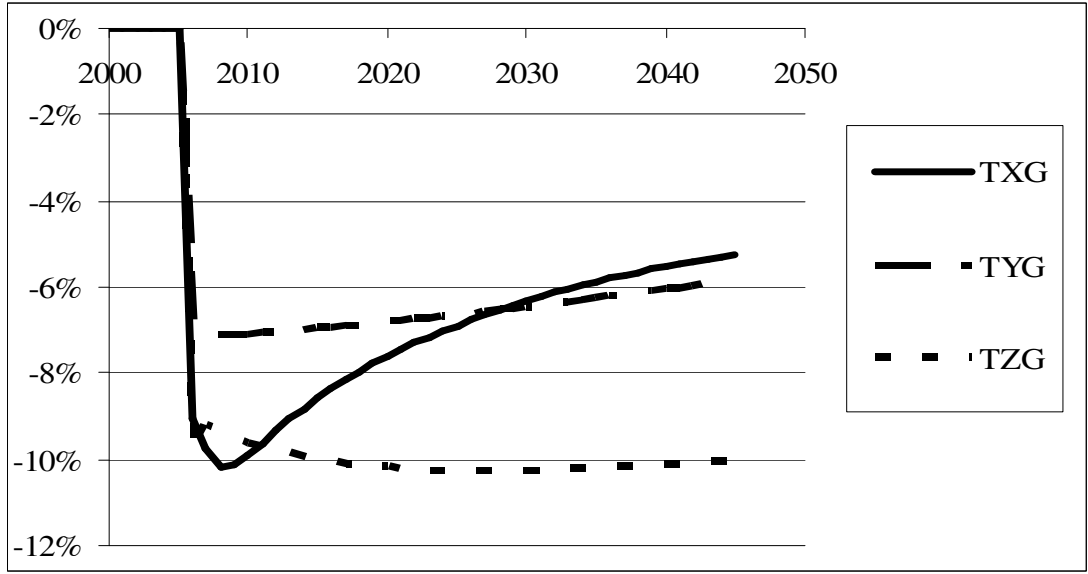

Source: estimations by model II

Figure 7 shows that tax revenue of regional budget in absolute prices is decreased. 


\section{CONCLUSIONS}

The technology of model external parameter estimation offered in work is based on use of high-speed parallel calculations on multiprocessing systems, reduction of number of independent parameters due to the assumption of regional economy equilibrium in the initial moment of calculation and a grouping of parameters by their economic sense.

Numerical experiments with the identified model of regional economy have shown, that dynamics of macroindexes of Kirov Region economy essentially depends on the policy spent by the regional government.

The model constructed here is an innovative product and the experience received on its parameter estimation is those "know-how" which can be used in the adaptation of the given model for concrete regional economic systems. Estimated values of the parameters give new knowledge about the macroeconomic system under investigation. This knowledge forces a researcher to modify the model. Thus, macroeconomic model parameter estimation based on parallel processing becomes a powerful tool for mathematical modelling of economic systems. These type models of regional economies can be used to study trade interactions of regions

The integration scenario calculated by numerical experiments with presented model convincingly shows that reduction of the customs duties as a result of integration increases outputs of production sectors, reduces a price level, and gives the increase of competitive advantages of region.

The work is in part supported by the Russian Humanitarian Scientific Foundation (Grant 06-02-91821); by the Russian Foundation of Basic Research (Grant 07-0100564); by the Program of State Support of Leading Scientific Schools (Grant SS5379.2006.1); and by the Program of Basic Research no.15 of Presidium of the Russian Academy of Sciences.

\section{REFERENCES}

Burnaev E.V., Olenev N.N., Starikov A.S. (2007) Parameter Estimation of a Macroeconomic Model. Proceedings of V Moscow International Conference on Operation Research (ORM2007) dedicated to the outstanding Russian scientist Nikita N. Moiseev $90^{\text {th }}$ birthday. Moscow. 71-73. 